\title{
Effective Diffusivity of Gas through Coke*
}

\section{By Yoshihito SHIGENO,** Saburo KOBAYASHI** and Yasuo OMORI**}

\begin{abstract}
Synopsis
In order to evaluate the effective diffusivity (De) for $\mathrm{CO}$ and $\mathrm{CO}_{2}$ through coke at elevated temperatures, two sorts of experiments based on the following two methods were undertaken:

(1) Determination of De from direct measurement of the diffusion fux by the Wicke-Kallenbach method at ambient temperature

(2) Derivations of De by the use of a mathematical model from the overall oxidation reaction rate of coke with $\mathrm{CO}_{2}$.

The values obtained by those two methods were compared with each other and it was found that they were in a fairly good accordance. It suggests that the overall reaction rate can be predicted by the use of $D e$ obtained by the independent measurement (1).
\end{abstract}

Key words: coke; gas diffusivity; carbon oxides; mathematical model; oxidation rate of coke.

\section{Introduction}

The quality of coke, such as strength and reactivity is one of the most significant factors influencing the performance of blast furnace operation. ${ }^{1)}$ Especially, the solution loss reaction in the shaft portion of the blast furnace decreases the strength of coke. Its reaction rate is principally governed by the diffusion rates of $\mathrm{CO}$ and $\mathrm{CO}_{2}$ through the coke; therefore Turkdogan et al. ${ }^{2}$ ) originally studied the gaseous diffusion through coke by means of the canister method. This method is the unique one by which direct evaluation of $D e$ can be done at elevated temperatures. Nevertheless, it has some disadvantages, e.g., existence of the inevitable difference between the total pressures inside and outside of the canister vessel when the molecular weights of diffusing gases are not equal*** and lack of capability of distinguishing among the Knudsen, normal and surface diffusion. On the other hand, the Wicke-Kallenbach method (W-K method) is the most basic one to evaluate $D e$ and has no disadvantages discribed above. The only problem to overcome, in the present stage, is its extension to the elevated temperatures.**** However this problem can be practically solved as long as the temperature dependence of $D e$ is known.

In the present study, to obtain $D e$ required in estimating the overall reaction rate of coke, the following two methods were employed. The flow diagram of the investigation is illustrated in Fig. 1. Method 1:

The value of $D e$ was evaluated from direct measurement of the diffusion fluxt by the $\mathrm{W}-\mathrm{K}$ method at ambient temperatures. In order to relate $D e$ evaluated at elevated temperatures by the subsequent method to this one at ambient temperatures, the temperature dependence of $D e$ was studied in the relatively low temperature range.

\section{Method 2:}

The overall reaction rate of oxidation of coke with $\mathrm{CO}_{2}$ was measured, and $D e$ was evaluated on the basis of a mathematical model. Then the value was extrapolated to that at ambient temperatures.

Finally, to clarify whether $D e$ evaluated by the method 1 can be used to estimate the overall reaction rate or not, the values obtained by those different two methods were compared with each other.

\section{Experimental Procedure}

1. Sample

Method 1:

The samples were restricted to formed coke for the sake of obtaining those without cracks across the specimens.

The lumpy coke was formed in the disk shape (20 $\mathrm{mm}$ in diameter and 2 to $4 \mathrm{~mm}$ in thickness). Some specimens were oxidized in $\mathrm{CO}_{2}$ to enlarge the pore volume. To make the pore distribution to be as homogeneous as possible through the sample, the

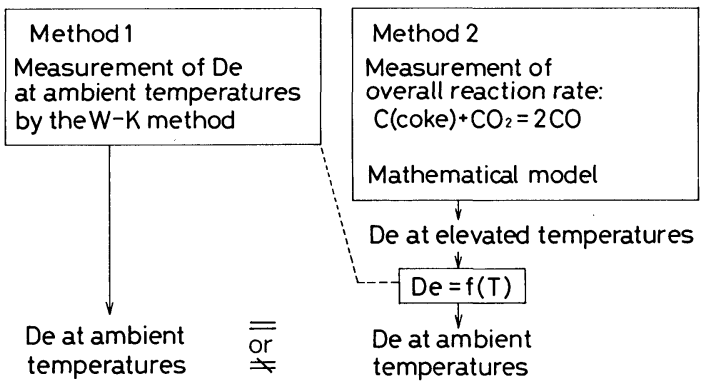

Fig. 1. Flow diagram of the present investigation.

* $\quad$ Based on the papers partly presented to the 107th ISIJ Meeting, April 1984, S55, at Chiba Institute of Technology in Narashino and partly published in Tetsu-to-Hagané, 63 (1977), 1081 and 64 (1978), 187, in Japanese.

This article was presented to the 4th International Symposium on Agglomeration, June 1985, in Toronto, by support of the Hyuga Fund of ISIJ. Printed with permission from Proceedings of the 4th International Symposium on Agglomeration, ed. by G. E. Capes, Iron and Steel Society of AIME, PA, (1985), 819.

Manuscript received on March 3, 1986; accepted in the final form on November 14, 1986. (C) 1987 ISIJ

** Research Institute of Mineral Dressing and Metallurgy, Tohoku University, Katahira, Sendai 980

*** The problems of such pressure difference are discussed by the authors elsewhere. ${ }^{3)}$

**** As noted in Chap. IV, the W-K method was improved to be adapted at elevated temperatures $(1573 \mathrm{~K})$ recently by the authors. $\uparrow \quad$ The term " diffusion flux" means the flux consisting of Knudsen, molecular and surface diffusion. 
oxidation was conducted at $973 \mathrm{~K}$.

Method 2:

Two kinds of coke (metallurgical as well as formed coke) were used; however the comparison of $D e$ with that by method 1 was carried out only for formed coke. They were shaped into a slab (30 mm in length and $30 \mathrm{~mm}$ in width) with several thickness from 1 to $5 \mathrm{~mm}$ for metallurgical coke and from 0.5 to $3.5 \mathrm{~mm}$ for formed coke. The properties of the samples are listed in Table 1.

\section{Apparatus and Procedure}

\section{Method 1:}

The experimental apparatus is illustrated in Fig. 2. Two kinds of gas systems, $\mathrm{CO}-\mathrm{CO}_{2}$ and $\mathrm{Ar}-\mathrm{He}$, were used. Coke specimen was mounted in a ring made of silicon rubber and was interposed between the two flanges of diffusion cell. One end face of specimen was exposed to a stream of $\mathrm{CO}$ (or Ar) and the other to a stream of $\mathrm{CO}_{2}$ (or He). $\mathrm{CO}$ (or Ar) diffusing through the pore and into the $\mathrm{CO}_{2}$ (or $\mathrm{He}$ ) stream and vice versa were analyzed by the gas chromatograph. From the concentrations and flow rates of gases, the diffusion flux for each component was determined. The temperature of the diffusion cell was controlled in the use of either a water bath regulated by a thermostat or an electric furnace over a wide range of temperatures. Temperature dependence of $D e$ was studied in a range from 273 to $423 \mathrm{~K}$.

Table 1. Averaged physical properties of coke.

\begin{tabular}{lcccc}
\hline Kind of coke & $\begin{array}{c}\text { Density } \\
\left(\mathrm{g} / \mathrm{cm}^{3}\right)\end{array}$ & $\begin{array}{c}\text { Apparent } \\
\text { density } \\
\left(\mathrm{g} / \mathrm{cm}^{3}\right)\end{array}$ & $\begin{array}{c}\text { Porosity } \\
(-)\end{array}$ & $\begin{array}{c}\text { Ash } \\
(\%)\end{array}$ \\
\hline $\begin{array}{l}\text { Metallurgical } \\
\text { coke }\end{array}$ & 1.94 & 0.94 & 0.52 & 10.8 \\
Formed coke & 1.94 & 1.30 & 0.33 & 9.9 \\
\hline
\end{tabular}

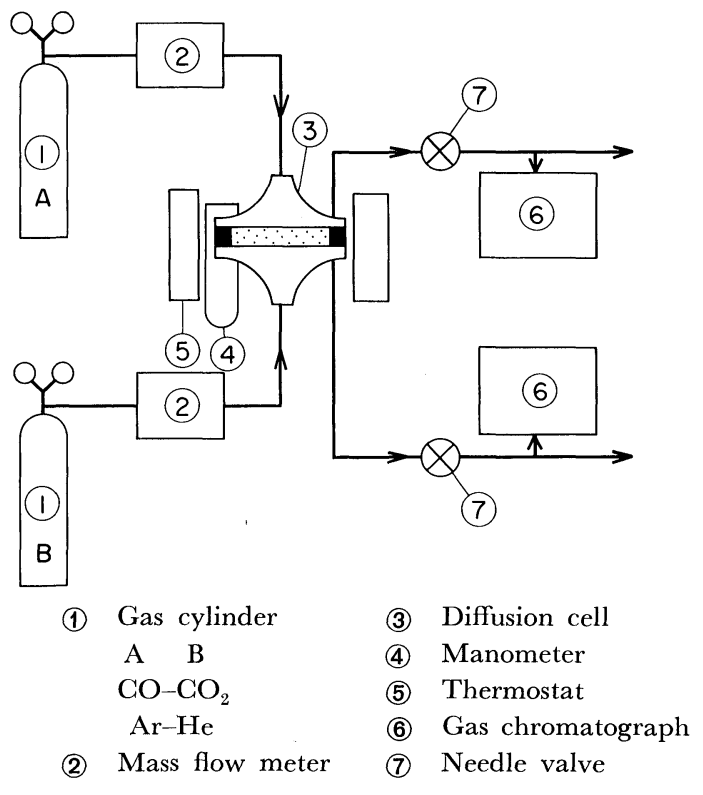

Fig. 2. Experimental apparatus for the Wicke-Kallenbach method.
Total fluxes of single gas system for $\mathrm{Ar}$ and $\mathrm{He}$ were also measured. From these measurements and diffusion flux for Ar-He gas system, parameters which are required to express the transport of gas through the porous media can be obtained. By the use of these parameters characteristic of the pore structure, Knudsen and surface diffusion as well as normal diffusion are evaluated separately.

Method 2:

The specimen was suspended by a platinum wire in the reaction tube made of alumina $(52 \mathrm{~mm}$ in inner diameter) as shown in Fig. 3. Carbon dioxide was introduced from the bottom of the furnace and preheated in the fixed bed of alumina spheres $(10 \mathrm{~mm}$ in diameter). Gas velocity on the surface of the specimen was forced to raise for decreasing the boundary layer resistance by the use of a slit in which the specimen was inserted during the reaction. The change in sample weight in the course of reaction was measured by a weighing devise; from these data the overall reaction rate was determined. By adapting these results to a general differential equation for oxidation of coke described in the latter paragraph, De for $\mathrm{CO}_{2}$ was obtained.

\section{Fundamental Equation}

\section{Method 1:}

The effect of boundary layer resistance to mass transfer on the surface of the samples decreases with increasing gas flow rate. In Fig. 4, the relation between diffusion flux and gas flow rate is presented for $\mathrm{He}-\mathrm{Ar}$ and $\mathrm{CO}-\mathrm{CO}_{2}$ systems, respectively. The invariable fluxes with gas flow rate were regarded as those without the effect of boundary layer resistance. The ratios of diffusion fluxes of the two gas systems are also given in Fig. 4.

According to Evans et al. ${ }^{4)}$ or Scott and Dullien, ${ }^{5)}$

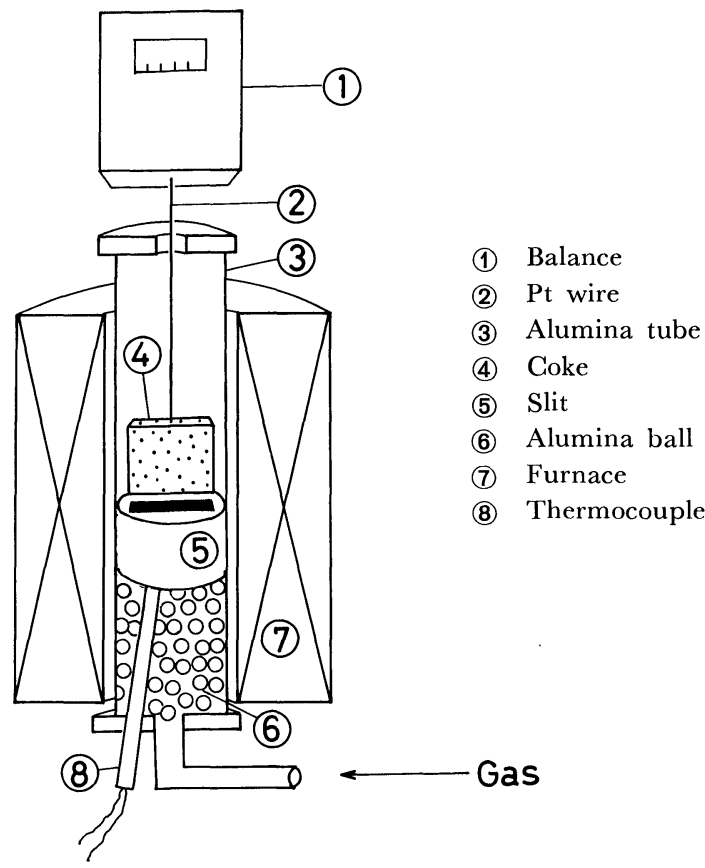

Fig. 3. Schematic diagram of experimental apparatus for oxidation of coke with $\mathrm{CO}-\mathrm{CO}_{2}$ gas mixtures. 


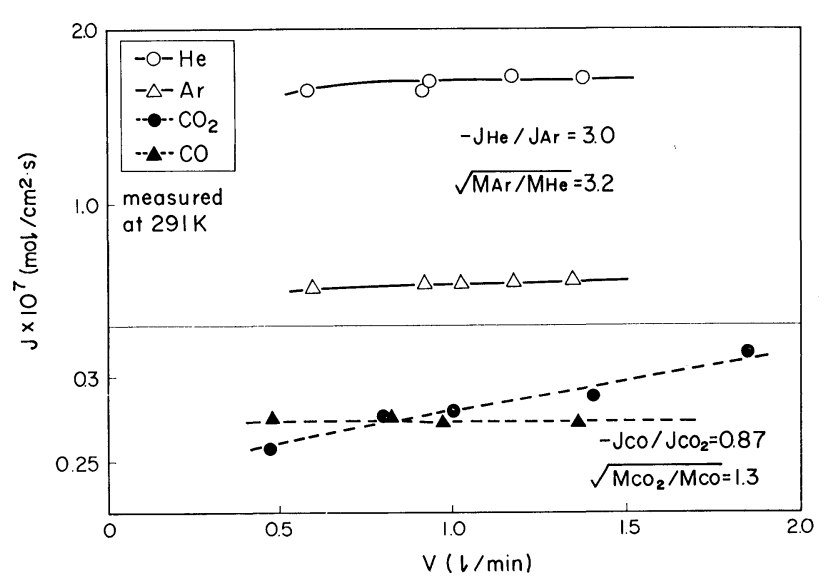

Fig. 4. Relation between diffusion flux through formed coke and flow rate of gas.

the following relation "the square root law" is proved to be adapted to the bimolecular gaseous diffusions from the Knudsen to the normal range.

$$
-J_{A} / J_{B}=\sqrt{ } \overline{M_{B} / M_{A}}
$$

For the Ar-He system, the square root law is satisfied within the range of experimental error; experimental value of $-J_{\mathrm{He}} / J_{\mathrm{Ar}}$ is 3.0 and theoretical one is 3.16. However, in the case of $\mathrm{CO}-\mathrm{CO}_{2}$ system, the ratio of gas fluxes deviates from that law; experimental value of $-J_{\mathrm{Co}} / J_{\mathrm{CO}_{2}}$ is 0.87 while the theoretical one is 1.25. The reason for this inconsistency is discussed in the latter paragraph.

Generally, the equation for the diffusion of component $A$ of the binary gases $A-B$ is expressed in Eq. $(2) .{ }^{4,5)}$ However, surface diffusion is excluded to avoid the complexity and it is discussed in Sec. V. 2.

$$
\begin{aligned}
J_{A}= & -\frac{c_{2} D_{A B} K_{A} P}{\left(K_{A}+c_{2} D_{A B}\right) R T} \nabla y_{A} \\
& +\frac{y_{A} K_{A}}{K_{A}+c_{2} D_{A B}}\left(J_{A}+I_{B}\right)
\end{aligned}
$$

where, $D_{A B}$ : binary free gas mutual diffusivity

$K_{A}$ : Knudsen diffusivity $\left(=c_{1} \sqrt{R T} / \overline{M_{A}}\right.$, $c_{1}$ : relatively Knudsen flow permeability)

$c_{2}$ : ratio of free gas mutual diffusivity within the porous medium to that in the space.

The parameters $c_{1}, c_{2}$ together with $c_{0}$ in Eq. (3) are geometric factors which are functions of the structure of the porous body, but are independent of the diffusing fluids. They are the basic parameters by which the transport of gas can be evaluated under any condition.

According to Gunn and King, ${ }^{6)}$ the total flux of gas component $\mathrm{A}$ is expressed as

$$
\begin{aligned}
\mathcal{N}_{A}= & -\frac{c_{2} D_{A B} K_{A} P}{\left(K_{m}+c_{2} D_{A B}\right) R T} \nabla y_{A} \\
& -\left[\frac{K_{A}\left(c_{2} D_{A B}+K_{B}\right)}{\left(c_{2} D_{A B}+K_{m}\right)}+\frac{c_{0} P}{\mu}\right] \frac{y_{A}}{R T} \nabla P .
\end{aligned}
$$

where, $c_{0}$ is relative D'Arcy flow permeability and $\mathcal{N} i$ is defined as total flux without surface diffusion, i.e., molecular and Knudsen diffusion flux plus hydrodynamic one, in distinction from $J_{i}$. Under the isobaric conditions, substitution of $\nabla P=0$ into Eq. (3) leads

$$
J_{A}=-\frac{c_{2} D_{A B} K_{A} P}{\left(c_{2} D_{A B}+K_{m}\right) R T} \nabla y_{A}
$$

By integrating the above equation under the boundary conditions $y_{A}=y_{A}^{0}$ at $z=0$ and $y_{A}=y_{A}^{L}$ at $z=L$, we obtain

$$
\frac{\left(y_{A}^{0} e^{\psi}-y_{A}^{L}\right)\left(1-\sqrt{\frac{M_{A}}{M_{B}}}\right)}{e^{\psi}-1}-1=\left(\frac{c_{2} D_{A B}^{0}}{K_{A} P}\right)
$$

where, $\quad \phi=J_{A} R T L(1-m) / c_{2} D_{A B} P$

$$
m=\sqrt{M_{A} / M_{B}}
$$

$D_{A B}^{0}=P D_{A B} \quad$ (constant value independent of total pressure).

Because the relation between the left-hand side of Eq. (5) and reciprocal of total pressure should designate a linear relation passing through the origin, several plots are made adjusting $c_{2}$ until a straight line is obtained. In this experiment, the pressure was varied from 1 to $4 \mathrm{~atm}$ and the optimum value of $c_{2}$ was found to be $1.6 \times 10^{-3}(-)$.

To evaluate $c_{0}$ and $c_{1}$, measurement of the total flux of single gas system is required. In such case, the conditions $\nabla y_{A}=0$ and $y_{A}=1$ hold and the integration of Eq. (3) under the boundary conditions: $P=P_{1}$ at $z=0$ and $P=P_{2}$ at $z=L$ leads to

$$
\frac{\mathcal{N} \sqrt{M R T}}{\Delta P}=\frac{c_{1}}{L}+\frac{c_{0}}{L^{2}}\left(\frac{\bar{P} L}{\mu} \sqrt{\frac{M}{R T}}\right)
$$

where, $\mathcal{N}$ : total flux of single gas system

$$
\begin{aligned}
& \bar{P}=\left(P_{1}+P_{2}\right) / 2 \\
& \Delta P=P_{1}-P_{2} .
\end{aligned}
$$

Equation (6) suggests that $\mathcal{N}$ is directly proportional to $\triangle P$ in the range where the variation of $\bar{P}$ is neglected. Such relation is shown in Fig. 5, and the slope is different depending on the molecular weight of gas species. By the use of two kinds of inert gases such as argon and helium, two relations between $c_{0}$ and $c_{1}$ can be obtained. In consequence, the solutions for these equations are $c_{0}=6.7 \times 10^{-9}\left(\mathrm{~cm}^{2}\right)$ and $c_{1}=$ $1.4 \times 10^{-4}(\mathrm{~cm})$, where the free gas mutual diffusivity and viscosity at $291 \mathrm{~K}$ used for these calculations are as follows : $D_{\mathrm{He}-\mathrm{Ar}}=0.701\left(\mathrm{~cm}^{2} / \mathrm{s}\right), \mu_{\mathrm{Ar}}=2.21 \times 10^{-4}$ $(\mathrm{g} / \mathrm{cm} \cdot \mathrm{s})$ and $\mu_{\mathrm{He}}=1.94 \times 10^{-4}(\mathrm{~g} / \mathrm{cm} \cdot \mathrm{s})$. These values were evaluated by using the Hirschfelder's formulas for diffusion and viscosity, respectively.

By the use of the values obtained above, it can be determined whether the Knudsen or normal diffusion prevails. The following expressions from the first and the second terms in Eq. (2) are rewritten respectively as

$$
\frac{c_{2} D_{A B} K_{A}}{K_{A}+c_{2} D_{A B}}=c_{2} D_{A B} /\left(1+\frac{c_{2} D_{A B}}{K_{A}}\right)
$$




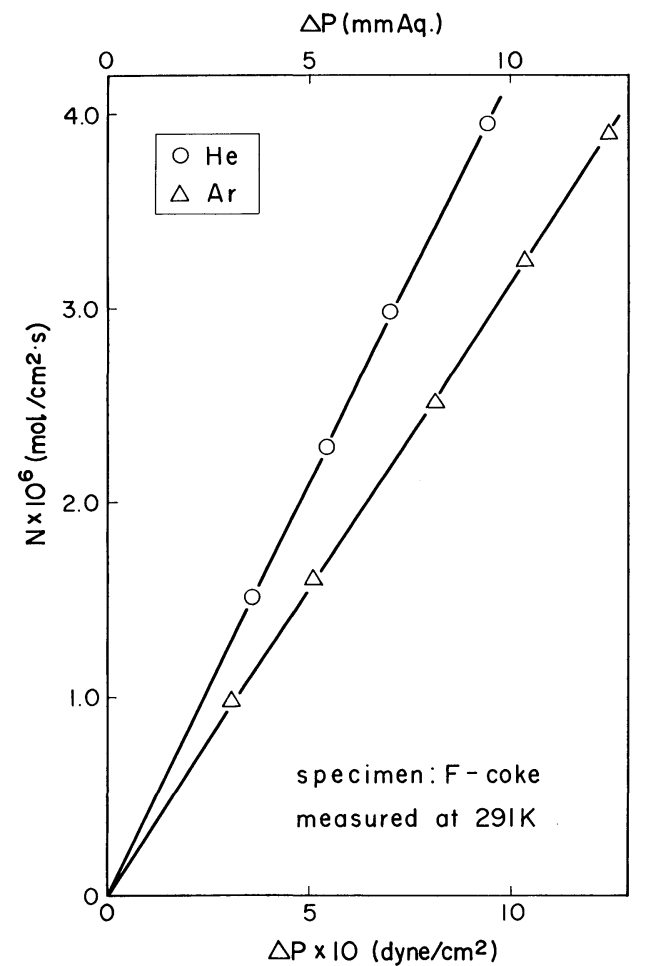

Fig. 5. Relation between total flux of single gas system and pressure difference.

$$
\frac{y_{A} K_{A}}{K_{A}+c_{2} D_{A B}}=y_{A} /\left(1+\frac{c_{2} D_{A B}}{K_{A}}\right)
$$

The ratio of $c_{2} D_{A B} / K_{A}$ represents the relative magnitude of Knudsen and normal diffusion; they are $1.0 \times 10^{-4}$ for helium and $2.9 \times 10^{-4}$ for argon. Therefore both values are negligibly small in comparison with unity. At elevated temperatures, this ratio may increase, e.g., at $1300 \mathrm{~K}$, it is about six times as that at $273 \mathrm{~K}$, which also can be ignored, though.

From this argument, Eq. (2) can be arranged into more simplified form as follows:

$$
J_{A}=-\frac{c_{2} D_{A B} P}{R T} \nabla y_{A}+y_{A}\left(J_{A}+J_{B}\right)
$$

Integration of Eq. (9) under the boundary conditions: $y_{A}=y_{A}^{0}$ at $z=0$ and $y_{A}=y_{A}^{L}$ at $z=L$ leads to

$$
J_{A}=\frac{c_{2} D_{A B} P}{R T L \alpha} \ln \frac{1-\alpha y_{A}^{L}}{1-\alpha y_{A}^{0}}
$$

From Eq. (10), $D e\left(=c_{2} D\right)$ is obtained by substituting the measured volues of $J_{A}$ and $J_{B}$ under the isobaric conditions.

Method 2:

Variation of fractional weight loss of metallurgical coke with time is shown in Fig. 6, where in the early stages of oxidation, e.g., less than twenty per cent weight loss, deviation from linearity is not perceptible. The slope of the lines divided by the atomic weight of carbon, i.e.,

$$
R_{m}=-(d W / d t) / 12 W_{0}
$$

is taken as the rate in terms of decrease in atomic

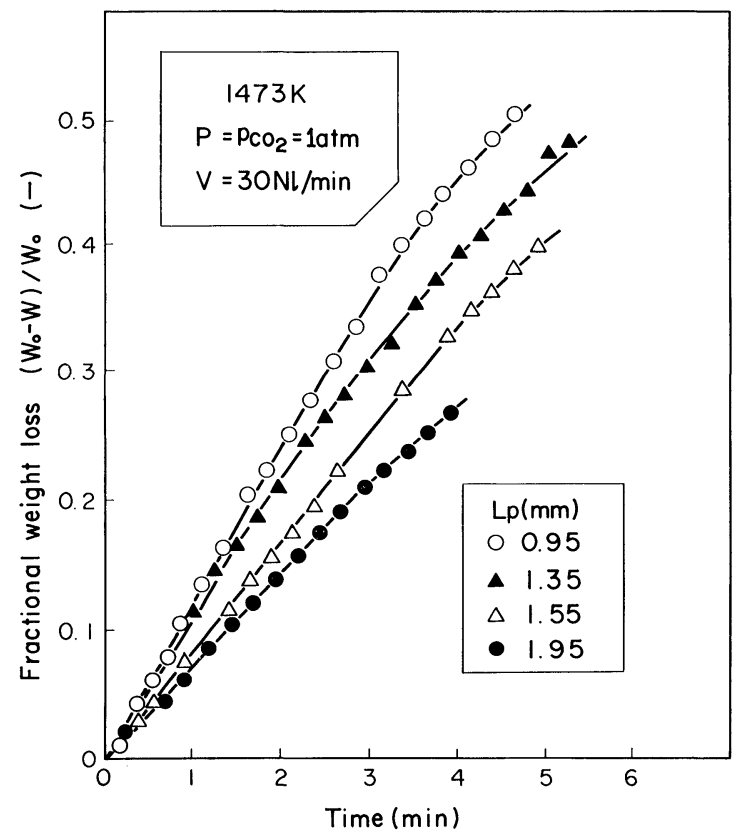

Fig. 6. Oxidation rate of metallurgical coke.

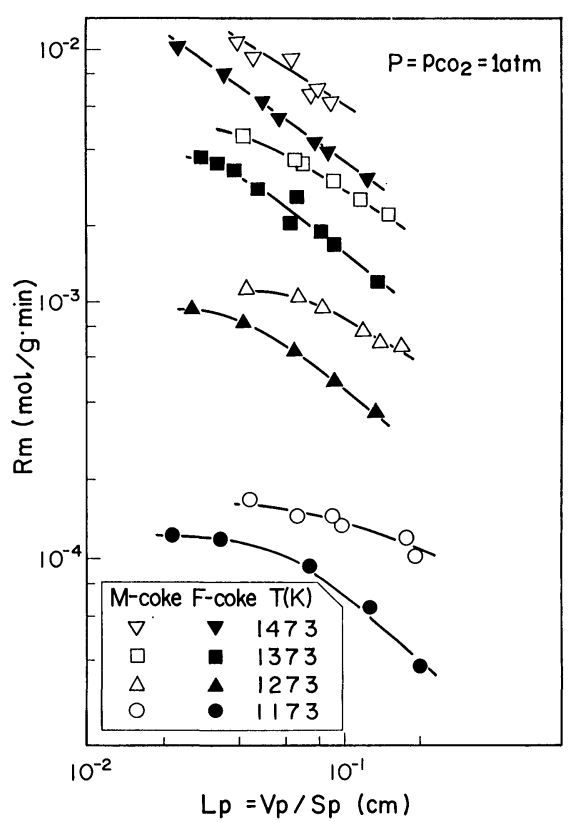

Fig. 7. Size effect on oxidation rate of slab samples.

carbon per unit time.

Under incomplete internal reaction conditions, the oxidation reaction rate is retarded more at the center of the specimen, i.e., there exists the profile of gas concentrations decreasing towards the center along the normal direction to the surface. Therefore, the overall reaction rate depends also on the thickness of the slab. Such size effect on the oxidation rate of formed coke is recognized in Fig. 7. The ratio of volume to outer surface area of slab, $V_{p} / S_{p}$ is taken as the characteristic thickness, which corresponds to the half of the thickness including the peripheral effect, though. The values of $V_{p}$ and $S_{p}$ were obtained by measuring its size. The general differential equation for oxidation of coke taking account 
of both internal reaction and diffusion of gas is given by as follows.9)

$$
\left(\nabla^{2} y\right) /(1+y)+\nabla y \cdot \nabla(1+y)^{-1}=\left(\rho_{a} R T / 60 P D e\right) R_{m}^{0}
$$

The reaction rate of oxidation of coke with $\mathrm{CO}-\mathrm{CO}_{2}$ gas mixtures in this equation, $R_{m}^{0}$, had been reported by the authors, ${ }^{10)}$ and the parameters contained in the "Langmuir type" rate equation were evaluated. As an example, that reported by Turkdogan and Vinters ${ }^{11}$ is represented as follows:

$$
R_{m}^{0}=k_{1}\left(P_{\mathrm{CO}_{2}}-P_{\mathrm{CO}_{2}, e}\right) /\left(1+k_{2} P_{\mathrm{Co}}\right)
$$

The kinetic parameters $k_{1}$ and $k_{2}$ obtained by the authors ${ }^{10)}$ are as follows:

For metallurgical coke

$$
\begin{aligned}
& k_{1}=\exp \left[17.890-61.695 \times 10^{3} / R T\right] \\
& k_{2}=\exp \left[-7.737+24.153 \times 10^{3} / R T\right]
\end{aligned}
$$

For formed coke

$$
\begin{aligned}
& k_{1}=\exp \left[19.917-66.625 \times 10^{3} / R T\right] \\
& k_{2}=\exp \left[-5.022+19.093 \times 10^{3} / R T\right]
\end{aligned}
$$

The numerical calculation by the use of Eqs. (12) to (17) with taking account of the effect of boundary layer resistance gives the value of $D e$.

\section{Experimental Results}

\section{Method 1:}

In Fig. 8, the relation between $D e$ measured at $273 \mathrm{~K}$ and porosity of coke is illustrated, where $D e$ was calculated from Eq. (10). The open triangles denote the unreacted coke and filled ones the coke with larger pores prepared by oxidation. The relationship is expressed as

$$
D e=4.58 \times 10^{-2} \varepsilon-0.0133 .
$$

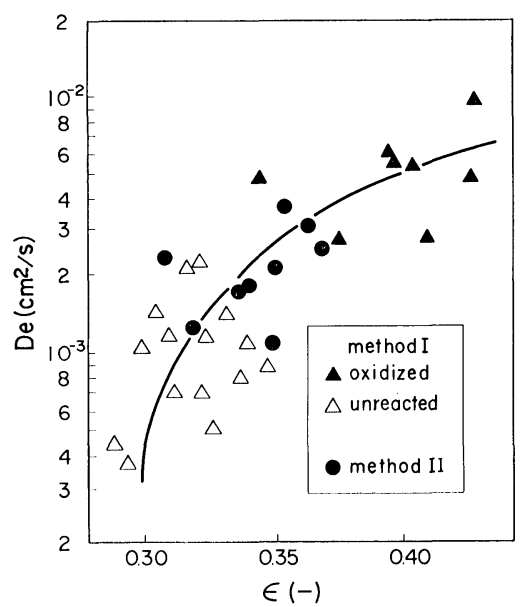

The effective diffusivities are the values at $273 \mathrm{~K}$.

- Extrapolated from the values at elevated temperatures shown in Fig. 10.

Fig. 8. Comparisons of effective diffusivities obtained by the Wicke-Kallenbach method (method 1) with those derived from the overall reaction rate (method 2). which can predict $D e$ of formed coke with a given porosity.

The relation between $D e$ and temperature was obtained from 273 to $423 \mathrm{~K}$ and presented in Fig. 9 by the filled circles. The porosity of the formed coke used in this experiment was 0.32 .

\section{Method 2:}

The values of $D e$ obtained at various temperatures are shown in Fig. 10 for both formed and metallurgical coke. Because the porosity of metallurgical coke is larger, $D e$ for metallurgical coke is about two times as large as that for formed coke.

\section{Discussion}

\section{Temperature Dependence of Effective Diffusivity for $\mathrm{CO}-\mathrm{CO}_{2}$ System}

As previously described, the Knudsen diffusion resistance is negligible, and therefore $D e$ should follow the theoretical temperature dependence in the normal diffusion range, i.e., $T^{1.5} \sim T^{2.0}{ }^{12)}$ The temperature dependence of $D e$ for $\mathrm{CO}-\mathrm{CO}_{2}$ system given in Fig. 9 shows that $D e$ certainly obeys $T^{1.5}$. In this figure, the results by Turkdogan et al. ${ }^{2}$ are referred, however, they were obtained by the use of canister method. Their effective diffusivities indicated by

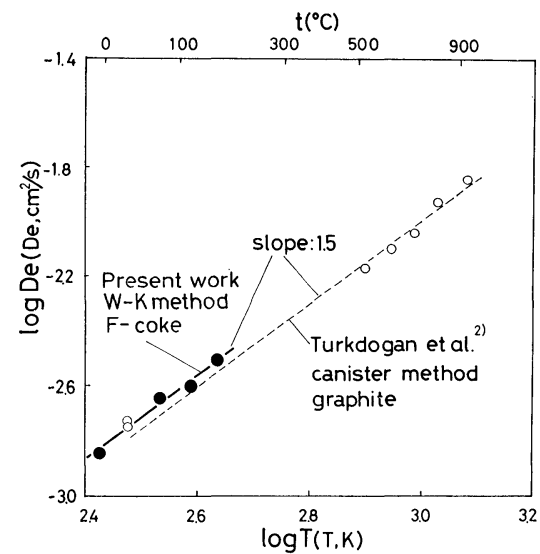

Fig. 9. Temperature dependence of effective diffusivities for $\mathrm{CO}-\mathrm{CO}_{2}$ system.

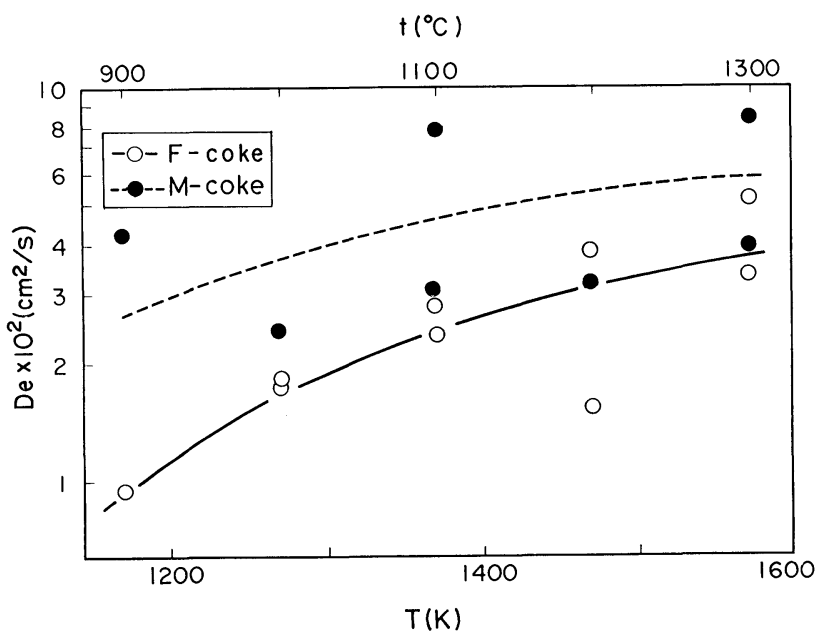

Fig. 10. Temperature dependence of the effective diffusivities for $\mathrm{CO}_{2}$ derived from overall reaction rates. 
the open circles with a dashed line also exhibit the temperature dependence of $T^{1.5}$. Consequently, such relation is considered to hold in the wide temperature range.

Recently, the authors developed the $\mathrm{W}-\mathrm{K}$ method into the more advanced one which can be adapted to higher temperatures (maximum temperature is $1573 \mathrm{~K}) .^{13)} \quad$ By this method, De for Ar-He system was confirmed to obey $\mathcal{T}^{1.5} \sim \mathcal{T}^{1.6}$ under the isobaric conditions from 294 to $1573 \mathrm{~K}$.

\section{Surface Diffusion}

In the previous section, the deviation of the ratio of diffusion fluxes from the "square root law" for $\mathrm{CO}-\mathrm{CO}_{2}$ system was noted (see Fig. 4). This deviation is considered to be attributed to the surface diffusion characteristic of adsorbable gas species, which occurs along with Knudsen and normal diffusions. By means of the $\mathrm{W}-\mathrm{K}$ method, the magnitude of surface diffusion can be evaluated, e.g., for the formed coke previously described $\left(c_{2}=1.6 \times 10^{-3}\right)$, $J_{\mathrm{CO}}$ and $J_{\mathrm{CO}_{2}}$ can be calculated from Eq. (10). The differences between the measured values and the calculated ones, $J_{\mathrm{CO}}^{\prime}-J_{\mathrm{CO}}$ and $J_{\mathrm{CO}_{2}}^{\prime}-J_{\mathrm{CO}_{2}}$ represent the fluxes of the surface diffusion, $J_{\mathrm{Co}}^{\mathrm{s}}$ and $J_{\mathrm{CO}_{2}}^{\mathrm{s}}$ respectively. The results are summarized in Table 2. According to Wicke and Kallebach ${ }^{14)}$ or Sakashita et al., ${ }^{15)}$ the effective diffusivity including surface diffusion is expressed as follows:

$$
D e=D e^{v}+D e^{s}
$$

where, $D e^{v}$ : the effective diffusivity for normal and Knudsen diffusion in series

$D e^{s}$ : the effective diffusivity for surface diffusion.

Although the temperature dependence of the effective diffusivity for surface diffusion has not been investigated over a wide temperature range, the magnitude of the surface diffusion is considered to decrease with an increase in temperature ${ }^{16)}$; generally, it can be neglected above $600 \mathrm{~K}$. Hence, it is seen from Eq. (19) that the contribution of surface diffusion may decrease as the temperature rises. From this argument and from the fact that $J_{i}^{s} / J_{i}^{\prime}$ is not so large as compared with the scatter of the data due to the difference of pore structure, it is understood to be appropriate that $J_{i}^{\prime}$ is substituted into Eq. (10) in calculating $D e$, although $J_{i}$ should be used to evaluate $D e$ at elevated temperatures.

\section{Comparisons of Effective Diffusivities by Wicke-Kallen- bach Method with Those Obtained from the Overall Reaction Rate}

The values of $D e$ shown in Fig. 10 were extrapolated to the values at $273 \mathrm{~K}$ according to the proportionality of $D e$ to $T^{1.5}$ and replotted in Fig. 8 together with the results by method 1 . The values evaluated by the different two methods are in good accordance with each other within the range of scatter. In consequence, it is concluded that $D e$ by the $\mathrm{W}-\mathrm{K}$ method is available for estimating the overall reaction rate of
Table 2. Ratio of the flux of surface diffusion to the diffusion flux through the formed coke.

\begin{tabular}{|c|c|c|c|}
\hline Gas & $\begin{array}{l}\text { Diffusion flux } J_{i}^{\prime} \\
\quad\left(\mathrm{mol} / \mathrm{cm}^{2} \cdot \mathrm{s}\right)\end{array}$ & $\begin{array}{l}\text { Flux of surface } \\
\text { diffusion } J_{i}^{s} \\
\left(\mathrm{~mol} / \mathrm{cm}^{2} \cdot \mathrm{s}\right)\end{array}$ & $\frac{J_{i}^{s}}{J_{i}^{\prime}} \times 100(\%)$ \\
\hline $\mathrm{GO}$ & $2.5 \times 10^{-8}$ & $1.8 \times 10^{-9}$ & 7.2 \\
\hline $\mathrm{CO}_{2}$ & $2.3 \times 10^{-8}$ & $4.7 \times 10^{-9}$ & 20 \\
\hline
\end{tabular}

coke.

\section{Conclusions}

(1) The overall reaction rates of oxidation of coke with $\mathrm{CO}_{2}$ were measured for the slab specimens over a temperature range from 1173 to $1474 \mathrm{~K}$. By the use of the mathematical model taking account of both gas diffusion and internal reaction, the effective diffusivities for $\mathrm{CO}_{2}$ were determined.

(2) The effective diffusivities for $\mathrm{CO}-\mathrm{CO}_{2}$ through formed coke were obtained over a temperature range from 273 to $423 \mathrm{~K}$ by the use of the Wicke-Kallenbach method. In addition, to determine the structural parameters, the total flux of single gas system and diffusion flux of $\mathrm{Ar}-\mathrm{He}$ were measured. From these results, it was found that the Knudsen diffusion could be neglected and moreover the contributions of surface diffusion of $\mathrm{CO}$ and $\mathrm{CO}_{2}$ to the total flux were evaluated.

(3) The effective diffusion coefficients derived from the overall reaction rates were extrapolated to those at $273 \mathrm{~K}$ and were compared with the values obtained by the Wicke-Kallenbach method. They were agreed fairly well with each other, which showed that the effective diffusivity required to estimate the overall reaction rate of coke could be evaluated by the direct measurement of diffusion flux at ambient temperatures.

\section{Nomenclature}

$c_{0}$ : constant dependent only upon structure of porous medium and giving relative D'Arcy flow permeability $\left(\mathrm{cm}^{2}\right)$

$c_{1}$ : constant dependent only upon structure of porous medium and giving relative Knudsen flow permeability (cm)

$c_{2}$ : constant dependent only upon structure of porous medium and giving the ratio of free gas mutual diffusivity within the porous medium to that in the space (-)

$D_{A B}$ : binary free gas mutual diffusivity for system $A-B\left(\mathrm{~cm}^{2} / \mathrm{s}\right)$

$D e:$ effective diffusivity of gas $\left(\mathrm{cm}^{2} / \mathrm{s}\right)$

$D e^{s}$ : effective diffusivity of surface diffusion $\left(\mathrm{cm}^{2} / \mathrm{s}\right)$

$D e^{v}$ : effective diffusivity of molecular and Knudsen diffusion $\left(\mathrm{cm}^{2} / \mathrm{s}\right)$

$J_{A}, J_{B}$ : diffusion flux of $A$ and $B$ without surface diffusion of the binary gas system $\left(\mathrm{mol} / \mathrm{cm}^{2} \cdot \mathrm{s}\right)$

$J_{\mathrm{Co}}^{\prime}, J_{\mathrm{CO}_{2}}^{\prime}$ : diffusion flux of $\mathrm{CO}$ and $\mathrm{CO}_{2}$ (including surface diffusion) $\left(\mathrm{mol} / \mathrm{cm}^{2} \cdot \mathrm{s}\right)$

$J_{\mathrm{CO}}^{\mathrm{s}}, J_{\mathrm{CO}_{2}}^{\mathrm{s}}$ : flux of surface diffusion of $\mathrm{CO}$ and $\mathrm{CO}_{2}$ 
$\left(\mathrm{mol} / \mathrm{cm}^{2} \cdot \mathrm{s}\right)$

$K_{A}, K_{B}: \quad$ Knudsen diffusivity $=c_{1} \sqrt{ } R T / M_{i}\left(\mathrm{~cm}^{2} / \mathrm{s}\right)$

$K m=y_{A} K_{B}+y_{B} K_{A}\left(\mathrm{~cm}^{2} / \mathrm{s}\right)$

$k_{1}$ : reaction rate constant of a Langmuir type rate equation $(\mathrm{mol} / \mathrm{g} \cdot \mathrm{min} \cdot \mathrm{atm})$

$k_{2}$ : adsorption equilibrium constant of a Langmuir type rate equation (1/atm)

$L:$ sample thickness $(\mathrm{cm})$

$L_{p}=V_{p} / S_{p}$

$M:$ molecular weight $(\mathrm{g} / \mathrm{mol})$

$\mathcal{N}:$ total flux of single gas system $\left(\mathrm{mol} / \mathrm{cm}^{2} \cdot \mathrm{s}\right)$

$\mathcal{N}_{A}, \mathcal{N}_{B}$ : total flux of $A$ and $B$ of the binary gas system $\left(\mathrm{mol} / \mathrm{cm}^{2} \cdot \mathrm{s}\right)$

$P: \quad$ total pressure $(\mathrm{atm})$

$\Delta P:$ difference of the total pressures (atm)

$\bar{P}:$ arithmetic mean of total pressures (atm)

$P_{\mathrm{CO}}, P_{\mathrm{CO}_{2}}:$ partial pressure of $\mathrm{CO}$ and $\mathrm{CO}_{2}(\mathrm{~atm})$

$P_{\mathrm{Co}, e}$ : partial pressure of $\mathrm{GO}$ in equilibrium with carbon for a particular value of $P_{\mathrm{CO}}(\mathrm{atm})$

$R:$ gas constant $\left(=8.31 \times 10^{7} \mathrm{erg} / \mathrm{mol} \cdot \mathrm{deg}\right)$

$R_{m}$ : overall reaction rate $(\mathrm{mol} / \mathrm{g} \cdot \mathrm{min})$

$R_{m}^{0}$ : reaction rate $(\mathrm{mol} / \mathrm{g} \cdot \mathrm{min})$

$S_{p}:$ outer surface area of specimen $\left(\mathrm{cm}^{2}\right)$

T: temperature $(\mathrm{K})$

$t$ : time (min)

$V:$ flow rate $(\mathrm{Nl} / \mathrm{min})$

$V_{p}:$ volume of specimen $\left(\mathrm{cm}^{3}\right)$

$W: \quad$ weight of specimen $(\mathrm{g})$

$W_{0}$ : initial weight of specimen $(\mathrm{g})$

$y_{A}, y_{B}$ : mole fraction of gas component $A$ and $B(-)$

$z:$ vertical distance from the surface of specimen $(\mathrm{cm})$

$\rho_{a}:$ apparent density of specimen $\left(\mathrm{g} / \mathrm{cm}^{3}\right)$

$\mu:$ viscosity of gas $(\mathrm{g} / \mathrm{cm} \cdot \mathrm{s})$

$\varepsilon$ : porosity (-)

$$
\alpha: \equiv 1+J_{B} / J_{A}
$$

\section{Acknowledgements}

The authors wish to thank Mr. K. Takeda, Kawasaki Steel Corp., and Mr. H. Goto, Mitsui Mining and Smelting Co., Ltd., for carrying out a part of the experiment and also thank Sumitomo Metal Industries, Ltd. for providing the coke.

\section{REFERENCES}

1) N. Nakamura, Y. Togino and M. Tateoka: Ironmaking Steelmaking, 5 (1978), 1.

2) E. T. Turkdogan, R. G. Olsson and J. V. Vinters: Carbon, 8 (1970), 545.

3) R. Takahashi, A. Kani, J. Yagi and Y. Omori: Bull. Inst. Min. Dress. Met. Tohoku Univ., 39 (1983), 14.

4) R. B. Evans, III, G. M. Watson and E. A. Mason: J. Chem. Phys., 35 (1961), 2076.

5) D. S. Scott and F.A.L. Dullien: AIChEJ, 8 (1962), 113.

6) R. D. Gunn and C. J. King: AIChEJ, 15 (1969), 507.

7) R. B. Bird, W. E. Stewart and E. N. Lightfoot: Transport Phenomena, John Wiley \& Sons, New York, (1960), 511.

8) R. B. Bird, W. E. Stewart and E. N. Lightfoot: Transport Phenomena, John Wiley \& Sons, New York, (1960), 23.

9) S. Kobayashi and Y. Omori: Tetsu-to-Hagané, 64 (1978), 187.

10) S. Kobayashi and Y. Omori: Tetsu-to-Hagané, 63 (1977), 1081.

11) E. T. Turkdogan and J. V. Vinters: Carbon, 8 (1970), 39

12) T. R. Marrero, E. A. Mason: J. Phys. Chem. Ref. Data, 1 (1972), 3.

13) Y. Shigeno, S. Kobayashi and Y. Omori: Bull. Japan Inst. Metals, 25 (1986), 96.

14) E. Wicke and R. Kallenbach: Kolloid-Z., 2 (1941), 135.

15) K. Sakashita, H. Arai and H. Kobayashi: J. Chem. Eng. Japan, 31 (1967), 920.

16) D. M. Ruthven: Principles of Adsorption and Adsorption Processes, John Wiley \& Sons, New York, (1984), 137. 